\title{
Galaxy Formation with Hot Dark Mattor and Cosmic Strings
}

\author{
Robert H. Brandenberger* \\ Department of Physics \\ Brown University \\ Providence, R.I. 02912, USA
}

Hot dark matter particles have large thermal velocities at $t$ and hence cannot be gravitationally bound on small scales (free streaming). In models of formation of structure based on linear adiabatic perturbations all inhomogeneities on scales smaller than the maximal free streaming length $\lambda_{J}$ are washed out. The mass $M_{J}$ inside a ball of radius $\lambda_{J}$ exceeds the galaxy mass. Hence in the above models galaxies can only form by fragmentation of larger-scale objects. This is a severe problem.

The main result of recent work ${ }^{1,2}$ ) is that cosmic string loops which seed galaxies survive neutrino free streaming. By the time the loops decay the free streaming length has fallen below the galactic scale. enabling galaxies to form independently of clusters.

We have studied the dissipationless clustering ${ }^{3}$ ) of neutrinos (with a mass chosen to produce $\Omega=1$ ) about a seed loop. Starting from the Liouville equation for the phase space density we derived an integral equation for the neutrino energy density perturbation in Fourier space 1 . After numerically solving this equation for a spectrum of fourier modes we obtain the following mass profile about a seed loop of mass $M_{1} \cdot O M(r)$ is the mass accreted inside a ball of radius $r$.

$$
\delta M(r)=\frac{3}{2} M_{1} z_{e q}\left(1-\left(1+\frac{r}{I}\right) e^{-r / L}\right\} \text { with } L \cong 8 h_{50}-2 \mathrm{Mpc}
$$

We note that $L$ is significantly smaller than the maximal Jeans length $\lambda_{J}$ in an adiabatic model. For $r \ll L$ we get

$$
\sigma M(r) \cong \frac{3}{4} M_{1} z_{\text {eq }}\left(\frac{r}{L}\right)^{2}
$$

The basic scenarios of structure formation are identical in cosmic string models with hot and cold dark matter. In both cases the largest loops at $t$ seed clusters. smaller ones galaxies. The radius of the seed of a cosmic structure is determined by requiring the correct number density. The number density of loops of radii $R$ is given by a well determined distribution $n(R, t)$ 


$$
n(R, t)=\nu R^{-5 / 2} t_{e q}^{1 / 2} t^{-2}, \quad R\left\langle t_{e q^{\prime}} t>t_{e q}, v \sim 10^{-2}\right.
$$

There are important differences in the detailed predictions of cosmic string models with hot versus those with cold dark matter. We obtain the following results 2).

1. Cluster formation is unchanged (hence the value for the mass per unit length $\mu$ is the same).

2. The density profile (2) leads to flat galaxy halo rotation curves. With CDM we get $v(r) \sim r^{-1 / 8}$.

3. The mass function of galaxies is less steep with HDM than with CDM and is in good agreement with th ${ }_{2}$ Schechter luminosity functign for $M<10^{14} M_{0}$. We $g e t h(M) \sim M^{-3 / 2}$ compared to $n(M) \sim M^{-5 / 2}$ for CDM.

4. $n(M)$ asymptotically approaches $n(M) \sim M^{-1 / 2}$ for $M<<M$ where $M_{C U} \simeq 10 M_{0}$. The corresponding cutoff mass for CDM is lower.

5. Galaxy masses are - $P$ wer than with CDM. For objects with the mean separation of loh $_{50}$ Mpc we get a mass

$$
M \simeq 5.10^{10} M_{0} h_{50}^{6}\left[\frac{\sigma_{C}}{700}\right]^{8} c
$$

where $\sigma_{c}$ is the cluster velocity dispersion in $\mathrm{kms}^{-1}$ and $\mathrm{c}$ is a constant of order 1 which depends on $v$ and on the time of formation of cluster loops. The above takes into account loop decay and baryon accretion (we assume $\Omega_{B}=1 / 8$ ).

We conclude that a theory with hot dark matter and cosmic strings is a viable cosmological mggel. This conclusion has also been reached by Bertschinger and Watts

* Also at DAMTP. University of Cambridge. Cambridge CB3 9EW. UK.

1) R. Brandenberger. N. Kaiser and N. Turok. 'Dissipationless clustering of neutrino perturbations about a cosmic string loop'. Phys. Rev. D. in press (1987).

2) R. Brandenberger. N. Kaiser, D. Schramm and N. Turok. 'Galaxy and structure formation with hot dark matter and cosmic strings'. DAMTP preprint. July 1987.

3) J. Bond and A. Szalay. Ap. J. 274,443 (1984).

4) E. Bertschinger and P. Watts. MIT preprint (1987). 